\title{
Outcomes after Pediatric Fundoplication: Defining the Redo Population
}

\author{
Baerg $\mathrm{JE}^{*}$, Perrone $\mathrm{EE}^{1}$, Vannix $\mathrm{RA}^{1}$, Thorpe $\mathrm{DL}^{1}$, Gasior $\mathrm{A}^{2}$ and St Peter $\mathrm{SD}^{2}$ \\ ${ }^{1}$ Loma Linda University and Children's Hospital Loma Linda, CA, USA \\ ${ }^{2}$ Children's Mercy Hospital, Kansas City, MO, USA
}

${ }^{*}$ Corresponding author: Baerg JE, MD, Associate Professor, Pediatric Surgery, Rm. 21111, Coleman Pavilion, 11175 Campus St., Loma Linda, CA 92354, Fax: 909558 7978, Tel: 909558 4619, E-mail: jbaerg@llu.edu

Citation: Baerg JE, Perrone EE, Vannix RA, Thorpe DL, Gasior A, et al. (2015) Outcomes after Pediatric Fundoplication: Defining the Redo Population. J Paedatr Neonatal Dis 1(1): 102. doi: 10.15744/24565482.1 .102

\section{Received Date: April 28, 2015 Accepted Date: August 31, 2015 Published Date: September 02, 2015}

\begin{abstract}
Objective: The aims were to compare outcome variables in children with gastroesophageal reflux disease (GERD) and one Nissen fundoplication to children with redo fundoplications and define the pediatric redo population.

Methods: After IRB approval (\#5100277), a case control study was conducted of children younger than 18 years, from two children's hospitals, with one Nissen fundoplication (control group) or a redo performed between January 1995 and March 2011. Complete data were collected by phone calls to caregivers in December 2012. Only redo operations performed after recurrence of GERD symptoms and wrap herniation into the mediastinum confirmed by contrast radiograph were included. To define the redo population, variables present before initial fundoplication, and outcome variables identified in December 2012, were compared. Continuous variables were analyzed by t-tests and categorical variables by chi-square tests. Logistic regression evaluated variable independence.

Results: The families of 212 children were contacted (54\% male), 181 had one fundoplication (85.4\%) and 31 had redos (14.6\%). The median follow-up time for controls was 3.6 years (range: 0.9-16 years). The median time to first redo was 17 months (range: 1-108 months) and to second redo for 3/31 (9.7\%), it was 32.5 months (range: 23-69 months). A significantly greater number with redos, could not feed orally prior to undergoing their first Nissen fundoplication ( $\mathrm{p}=0.003)$. At follow-up evaluation, children with redos had significantly more hospital admissions for pneumonia $(\mathrm{p}=0.02)$, vomiting and retching $(\mathrm{p}=0.01)$, gastrojejunal feeding tubes $(\mathrm{p}=0.01)$ and wrap herniation on contrast radiograph $(\mathrm{p}=0.01)$. Logistic regression revealed vomiting, OR: 3.4 (95\% CI: 1.4-8.3) and retching, OR: 3.8 (95\% CI: 1.6-9.2) were independently associated with the redo population.
\end{abstract}

Conclusion: At follow-up evaluation, vomiting and retching are independent factors that define the redo population. Significantly more children with redos are fed by gastrojejunal feeding tubes because the redo fails to control their GERD symptoms.

Keywords: Redo Nissen fundoplication; Pediatric gastroesophageal reflux disease

List of abbreviations: GERD: Gastroesophageal reflux disease; ALTE: Acute life threatening event; BMI: Body mass index; UGI: upper gastrointestinal; GJ: Gastrojejunal

\section{Introduction}

Children with complications of gastroesophageal reflux disease (GERD) frequently undergo Nissen fundoplication. There is significant heterogeneity among pediatric GERD studies [1]. Surgical treatment for GERD is reported to improve quality of life, especially for neurologically impaired children [2,3]. Despite initial symptomatic relief, however, the incidence of redo operation after fundoplication is reported between 1.7 and 18 percent [3-6]. Little is known about the outcome for children with GERD that require a redo fundoplication. The aims of this study were to compare outcome variables in children with one Nissen fundoplication for gastroesophageal reflux disease (GERD) to those with redo operations, define the pediatric redo population and make clinical recommendations.

\section{Methods}

\section{Cohort}

After Institutional Review Board approval (5100277), a case control study was conducted of children younger than 18 years, from two children's hospitals, with Nissen fundoplications performed between January 1995 and March 2011. Variables for children with redo Nissen fundoplications were compared to those with one Nissen fundoplication to control their GERD symptoms (control group). 


\section{GERD Diagnosis}

Prior to undergoing initial fundoplication, all included children had failure of medical management and GERD complications such as failure to thrive, pneumonia or acute life threatening events (ALTEs). Surgeons made the decision for initial fundoplication according to center specific practice and the identification of clinical complications of GERD [7]. All included children had an upper gastrointestinal (UGI) contrast radiograph prior to their first fundoplication and 7 (3\%) had a hiatus hernia. There were 39 endoscopies and 13 (33\%) revealed esophagitis on biopsy. There were 60 esophageal pH monitoring studies, all were positive. At present, no single diagnostic study determines the severity of reflux in children [8].

Prior to redo fundoplication, all included children had recurrent GERD symptoms not responding to medical therapy and wrap transmigration on contrast radiograph.

Children with congenital diaphragmatic hernia or esophageal atresia were excluded. GERD is identified in up to half of children with either diagnosis and many undergo antireflux surgery, including more redo operations. Both have respiratory symptoms and failure to thrive that may or may not be attributable to GERD. Because GERD is diagnosed so frequently and the pathophysiology may differ, these diagnoses were excluded in order to limit comorbidities and strengthen the results with a more uniform cohort [9].

\section{Variables}

Variables identified prior to the initial Nissen fundoplication were: birth weight, gestational age, body-mass index (BMI)/weightlength z-score, age at first fundoplication, gender, neurological impairment, gestational age below 33 weeks, pneumonia, acute life threatening events (ALTEs), retching, failure to feed orally, congenital cardiac defects and chromosomal anomalies.

Children were considered neurologically impaired if irreversible brain damage existed prior to the initial fundoplication. Information was obtained from magnetic resonance imaging studies, head ultrasounds, hospital and clinic records. The BMI/ weight-length $\mathrm{z}$-scores were considered to reflect the nutritional status of the patients [10]. The World Health Organization (WHO) weight/length $\mathrm{z}$-scores were used to compare growth for children below two years of age and BMI z-scores for children above two years of age [11]. Children considered to have pneumonia had infection specific to the lungs and a chest radiograph consistent with pneumonia. Acute life-threatening events were defined as episodes characterized by a combination of apnea, color change, and a marked change in muscle tone, choking, or gagging [12]. Retching was defined as "a strong involuntary unproductive effort to vomit [13]." Children that could not feed orally were fed by gastrostomy or gavage feeding prior to fundoplication.

\section{Follow-up Evaluation}

Follow-up data were collected from hospital and clinic records to evaluate the status of the fundoplication at study end for survivors or at death for mortalities. Phone calls were made to assess the current state of health for children in December 2012 and collect outcome data. The cause of death was recorded from the hospital death summary. Only children with upper gastrointestinal (UGI) contrast radiographs at the time of follow-up evaluation were included. The results were reviewed for all 212 included children.

Outcome variables collected were: hospital admissions for pneumonia, vomiting, retching, state of fundoplication on UGI contrast radiographs, use of gastro-jejunal (GJ) feeding tube, laparotomies for adhesive bowel obstruction, ALTEs, a requirement for esophageal dilations, administration of anti-GERD medications at the time of follow-up phone call, and mortalities. Anti-GERD medications were defined as administration of H2-receptor antagonists or proton pump inhibitors [14]. The BMI weight/length $\mathrm{Z}$-scores at one year after fundoplication and at final follow-up evaluation were recorded.

\section{Surgery}

The operative technique of Nissen fundoplication, laparoscopic or open laparotomy, was reviewed and recorded for each case. In all, a standard Nissen fundoplication technique was performed and included takedown of the short gastric vessels, reduction of the stomach below the diaphragm, dissection and excision of any hernia sac, and closure of the crural defect posteriorly. A 360-degree fundal wrap was performed around the lower esophagus over a bougie dilator to ensure the wrap was loose. The wrap was secured to the diaphragm on each side of the crura [15].

\section{Analysis}

Variables were compared using t-tests for continuous and chi-square tests for categorical variables. Logistic regression evaluated for independence of variables and were expressed as odds ratios and $95 \%$ confidence intervals. A p-value less than 0.05 was considered significant. Means were expressed $+/$ - standard deviation and medians were expressed with ranges.

\section{Results}

The families of 212 children were contacted (53.8\% male), 181 had one fundoplication (85.4\%) and 31 had redos (14.6\%) (Figure 1). The median follow-up time for the cohort was 3.4 years (range: $0.5-16$ years) (mean: $4.1+/-2.9$ years). The median followup time for controls was 3.6 years (range: 0.9-16 years) (mean: $4.3+/-3.0$ years). The median time to first redo was 17.0 months (range: 1-108 months) (mean: 31.5 +/- 34.4 months). The median time to second redo after the first redo for 3/31(9.7\%) was 32.5 
months (range: 23-69 months) (mean: 41.5 +/- 24.3 months). One had a third redo five years after the second redo complicated by incarceration of small bowel in a para-esophageal hernia.

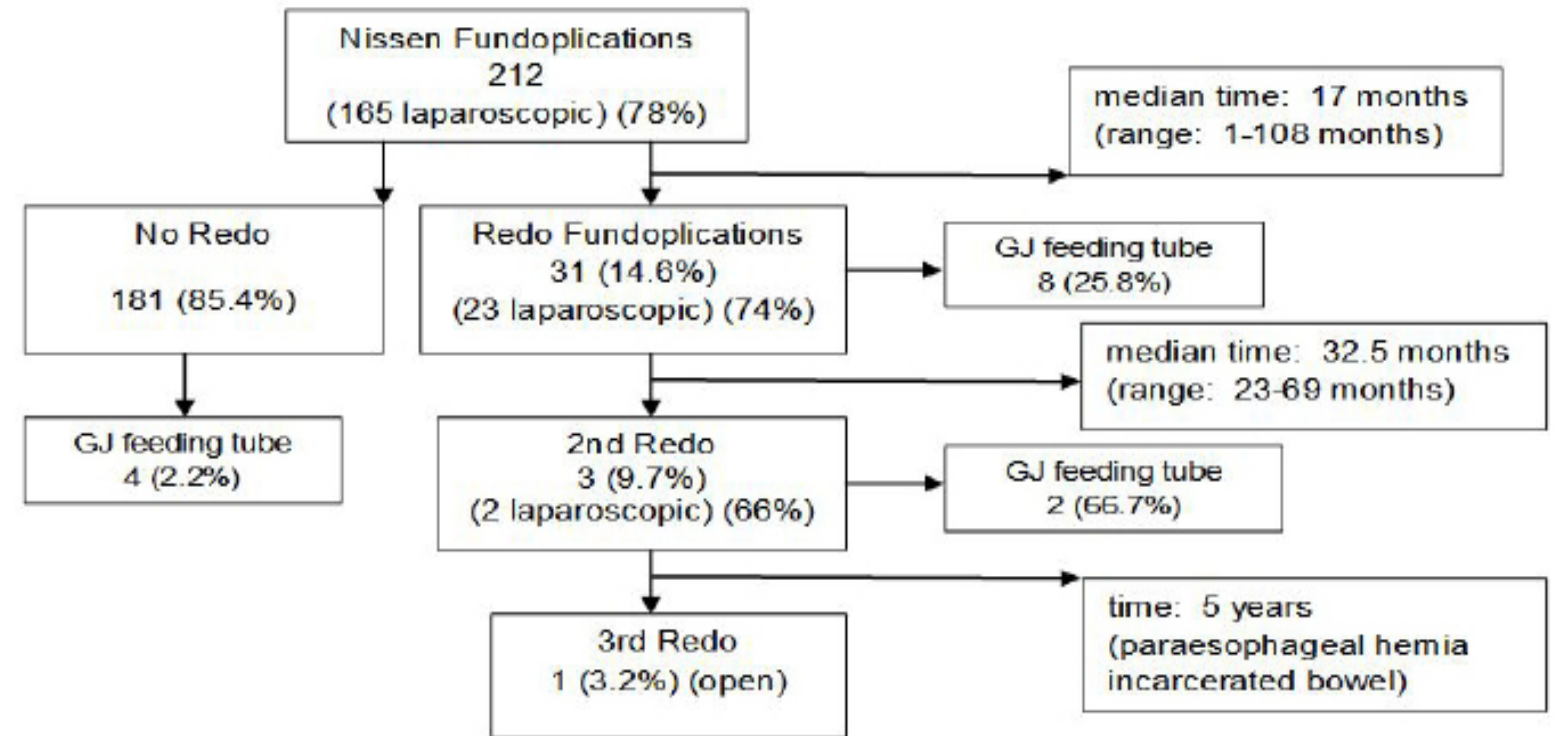

Figure 1: Time to redo Nissen fundoplication and distribution of gastro-jejunal (GJ) feeding tubes at final follow-up

During the follow-up period, 17/212 children (8.0\%) died. All deaths were due to respiratory failure from pneumonia at a median of 8.5 months (range: 14 days - 109 months) (mean: 19.4 +/- 46 months). The median time to death in the 3/17 (17.6\%) with redos was 31 months, range: 8.5 - 41 months (mean: 27 +/- 17 months). All underwent only one redo operation.

Factors present before the initial fundoplication in children with and without redo Nissen fundoplication, analyzed by univariate analysis are presented in Table 1 . There was a trend to statistical significance for younger patients to require a redo ( $\mathrm{p}=0.08$ ). The incidence of neurological impairment was similar for children with and without redos. However, a significantly greater number with redos, could not feed orally prior to undergoing their first Nissen fundoplication $(\mathrm{p}=0.003)$. No other variables identified prior to initial Nissen fundoplication were significant.

\begin{tabular}{|l|l|l|l|l|}
\hline Variable & $\begin{array}{l}\text { Cohort } \\
(\mathbf{2 1 2}\end{array}$ & $\begin{array}{l}\text { No Redo } \\
(\mathbf{1 8 1})\end{array}$ & $\begin{array}{l}\text { Redo } \\
(\mathbf{3 1})\end{array}$ & $\mathbf{p}^{\mathbf{2}}$ \\
\hline Birth weight (kg) & $2.5(1.8)$ & $2.4(1.0)$ & $2.8(1.0)$ & 0.28 \\
\hline Gestational age (weeks) & $35.6(5.1)$ & $35.4(5.2)$ & $36.4(4.4)$ & 0.30 \\
\hline BMI weight/length z-score & $-1.08(2.0)$ & $-1.04(2.1)$ & $-1.11(2.1)$ & 0.87 \\
\hline Age at first fundo (months) & $30.8(40.1)$ & $32.7(51.6)$ & $22.2(35.1)$ & 0.08 \\
\hline & & & & \\
\hline & $\mathbf{n}(\%)$ & $\mathbf{n}(\%)$ & $\mathbf{n ( \% )}$ & $\mathbf{p}^{\mathbf{3}}$ \\
\hline Gender (Male) & $114(54)$ & $96(53)$ & $18(58)$ & 0.60 \\
\hline Neurologic impairment & $137(65)$ & $115(64)$ & $22(71)$ & 0.42 \\
\hline Gestation <33 weeks & $56(26)$ & $50(28)$ & $6(19)$ & 0.33 \\
\hline Pneumonia & $52(25)$ & $42(23)$ & $10(32)$ & 0.28 \\
\hline ALTEs & $39(18)$ & $31(17)$ & $8(26)$ & 0.26 \\
\hline Retching & $111(52)$ & $95(53)$ & $16(52)$ & 0.93 \\
\hline Cannot feed orally & $98(46)$ & $76(42)$ & $22(71)$ & $\mathbf{0 . 0 0 3}$ \\
\hline Congenital cardiac defects & $50(24)$ & $43(24)$ & $7(23)$ & 0.87 \\
\hline Chromosomal anomalies & $74(35)$ & $66(36)$ & $8(26)$ & 0.25 \\
\hline
\end{tabular}

${ }^{1}$ Mean and standard deviation. Complete cohort used for calculations

${ }^{2}$ Student t-test

${ }^{3}$ Pearson chi-square test

Table 1: Variables present before first fundoplication 
Outcome variables for children with and without redos were analyzed by univariate analysis (Table 2). During the follow-up period, children with redo fundoplications had significantly greater hospital admissions for pneumonia $(\mathrm{p}=0.02)$ and symptoms of vomiting and retching $(\mathrm{p}=0.01)$. A significantly greater number with redos had wrap herniation on UGI radiographs ( $\mathrm{p}=0.01$ ), were fed by GJ feeding tubes ( $\mathrm{p}=0.01$ ) and more frequently required a laparotomy for adhesive bowel obstruction ( $\mathrm{p}=0.04)$.

\begin{tabular}{|l|l|l|l|l|}
\hline Variable & Cohort (212) $^{1}$ & No Redo (181) & Redo (31) & $\mathbf{p}^{\mathbf{2}}$ \\
\hline Pneumonia admissions & $39(18)$ & $28(16)$ & $11(36)$ & 0.02 \\
\hline Vomiting & $37(18)$ & $23(13)$ & $14(45)$ & 0.01 \\
\hline Retching & $39(18)$ & $24(13)$ & $15(48)$ & 0.01 \\
\hline Gastrojejunal feeding tube & $14(7)$ & $4(2)$ & $10(32)$ & 0.01 \\
\hline Bowel obstruction laparotomy & $6(3)$ & $3(2)$ & $3(10)$ & 0.04 \\
\hline ALTEs & $31 / 191(16)$ & $23(12)$ & $8(26)$ & 0.77 \\
\hline Dilations & $3(1)$ & $2(1.1)$ & $1(3)$ & 0.37 \\
\hline anti-GERD medications & $99(47)$ & $80(44)$ & $19(61)$ & 0.19 \\
\hline Death & $17(8)$ & $14(8)$ & $3(10)$ & 0.71 \\
\hline BMI weight/length z score & & & & $\mathbf{p}^{4}$ \\
\hline At first fundoplication & $-1.08(2.0)$ & $-1.04(2.1)$ & $-1.11(2.1)$ & 0.87 \\
\hline One year after fundoplication & $-0.14(1.9)$ & $-0.22(1.8)$ & $-0.03(2.2)$ & 0.19 \\
\hline At final evaluation & $-0.21(1.9)$ & $-0.18(1.9)$ & $-0.53(1.9)$ & 0.35 \\
\hline
\end{tabular}

$1(\%)$

${ }^{2}$ Pearson chi-square

${ }^{3}$ Mean and standard deviation. Complete cohort used for calculations unless indicated by a different denominator ${ }^{4}$ Student t-test

Table 2: Outcome variables identified on follow-up evaluation after fundoplication

The children who required laparotomy for obstruction were otherwise similar with $3 / 181$ (1.7\%) occuring after initial fundoplication and 3/31 (9.7\%) after redo operation. In each group, two of the initial fundoplications were laparoscopic and one was open.

At final follow-up evaluation, 8/181 (4\%) without redos, had an asymptomatic wrap herniation on UGI radiographs. The wrap was intact in the remainder.

The majority of the cohort (99/212) (47\%) received anti-GERD medications at study end. The incidence rose to $19 / 31$ (61\%) for those with redos but was not significantly higher $(\mathrm{p}=0.19)$. No other outcome variables were significant. Body mass index/weightlength z-scores increased for the cohort at one year after fundoplication, and final evaluation, but no significant differences were observed.

Logistic regression revealed vomiting, OR: 3.4 (95\% CI: 1.4-8.3) and retching, OR: 3.8 (95\% CI: 1.6-9.2) were independently associated with the redo population.

For children without redos, $83 \%$ (150/181) of parents contacted stated that GERD symptoms improved after fundoplication and they would proceed with fundoplication again compared to only $17 \%(5 / 31)$ of parents of children ith redos $(p=0.01)$.

\section{Discussion}

In this series of children with GERD undergoing one fundoplication or redo operation at a median of 3.4 years follow-up, the incidence of redo is $14.6 \%$ at a median of 17 months after the first operation. Ten percent with one redo undergo a second redo within three years. Others report similar failure rates between 12.5 and 20\%, and the rate increases in redos [3-6].

Similar to previous reports, vomiting and retching are independently associated with redo operation [16]. A significant number of children with redos are fed by gastrojejunal feeding tubes because surgery fails to address their feeding difficulties. They have significantly more hospital admissions for pneumonia.

Reports conflict regarding the association between neurologic impairment and redo operation [16,17]. Neurologic impairment alone does not increase the risk of redo [16]. The impairment, however, appears more profound in the redo population because children that will require redos are younger and most cannot feed orally. Profound impairment with spasticity at initial GERD presentation may predispose to vomiting and retching, early surgical consultation, tension on the wrap and eventual wrap failure.

In children with one fundoplication, phone calls to parents confirm GERD symptoms are controlled and $83 \%$ are satisfied with the operation. For those with redo fundoplications, satisfaction plummets to $17 \%$. Parents of the redo population indicate they would not proceed with fundoplication again.

Phone calls confirm the majority of the cohort receives anti-GERD medications at a median of 3.6 years follow-up evaluation. The redo population consumes the most [18]. Prescriptions are administered by ancillary physicians, therefore indications, symptoms, exact date of prescription and compliance are unknown. This information may promote more informed preoperative discussions in that surgery does not always ameliorate symptoms or feeding difficulties. 
The retrospective method is a limitation of this study. Some children may be lost to follow-up if they are not seeking treatment. However, the median follow-up for the control group exceeds the redos, which decreases the likelihood of missed cases. Surgeons perform fundoplication based on clinical criteria and center specific practice. $[1,7,8,19]$ To strengthen the analysis in this study, however, all included children have an UGI contrast study at follow-up evaluation. Only those who fail medical therapy and have wrap herniation on contrast radiograph prior to redo are included as redo fundoplications. The large multicenter cohort, and the fact that parents are directly contacted, counter balance the retrospective limitations.

The redo population is defined by continued vomiting and retching and redo operation is performed at a median of 17 months. After such early recurrence, one questions whether to proceed with a redo. Spastic, neurologically-impaired children with vomiting and retching, may benefit from the administration of Baclofen for symptom relief, before considering repeat operations [20]. Surgical options such as esophageal replacement, esophago-gastric dissociation or Roux-Y jejunal feeding tubes are reasonable options [21]. Gastrojejunal feeding tubes are a conservative option if surgical risks are unacceptable and are widely used [22].

Until specific investigations are instituted for all children suspected of GERD, both before initial fundoplication and at evaluation for wrap failure, questions will persist regarding fundoplication outcomes [1]. Similar to others, we found mortality after one fundoplication or redo is due to pneumonia and respiratory failure [23]. The redo population has a significantly higher incidence of admissions for pneumonia. Many attribute this to oropharyngeal dyscoordination, but videofluoroscopic swallowing studies are not routine [24].

To clarify surgical indications, we recommend identification of clinical complications of GERD from a detailed history [1,7,24]. Many surgeons consider that no single study diagnoses GERD. Children frequently undergo surgery for unclear indications, a consideration which our study supports $[1,7,8]$. We recommend a complete evaluation prior to proceeding with initial or redo fundoplication, including $\mathrm{pH}$ probe studies, endoscopy, contrast radiographs and videofluoroscopic swallowing studies [23,24]. This information may promote better selection of surgical options and inform discussions regarding prognosis, particularly in the redo population.

\section{References}

1. Martin K, Deshaies C, Emil S (2014) Outcomes of pediatric laparoscopic fundoplication: a critical review of the literature. Can J Gastroenterol Hepatol 28: 97-102.

2. Kristensen C, Avitsland T, Emblem R, Refsum S, Bjørnland K (2007) Satisfactory long-term results after Nissen fundoplication. Acta Paediatr 96: 702-5.

3. Kawahara H, Okuyama H, Kubota A, Oue T, Tazuke Y, et al. (2004) Can laparoscopic antireflux surgery improve the quality of life in children with neurologic and neuromuscular handicaps? J Pediatr Surg 39: 1761-4.

4. Pacilli M, Eaton S, McHoney M, Kiely EM, Drake DP, et al. (2014) Four year follow-up of a randomised controlled trial comparing open and laparoscopic Nissen fundoplication in children. Arch Dis Child 99: 516-21.

5. Kimber C, Kiely EM, Spitz L (1998) The failure rate of surgery for gastro-esophageal reflux disease. J Pediatr Surg 33: 64-6.

6. Diaz DM, Gibbons TE, Heiss K, Wulkan ML, Ricketts RR, et al. (2005) Anti-reflux surgery outcomes in pediatric gastroesophageal reflux disease. Am J Gastroenterolo 100: 1844-52.

7. Onyeador N, Paul SP, Sandhu BP (2014) Paediatric gastroesophageal reflux clinical practice guidelines. Arch Dis Child Educ Pract Ed 99: 190-3.

8. Rosen R, Levine P, Lewis J, Mitchell P, Nurko S (2010) Reflux events detected by pH-MII do not determine fundoplication outcome. J Pediatr Gastroenterolo Nutr 50: 251-5.

9. Lopez-Fernandez S, Hernandez F, Hernandez-Martin S, Dominguez E, Ortiz R, et al. (2014) Failed Nissen fundoplication in children: causes and management. Eur J Pediatr Surg 24: 79-82.

10. De Onis M, Garza C, Onyango AW, Rolland-Cachera MF (2009) WHO growth standards for infants and young children. Arch Pediatr 16: 47-53.

11. Meiz, Grummer-Strawn LM (2007) Standard deviation of anthropometric z-scores as a data quality assessment tool using the 2006 WHO growth standards: a cross country analysis. Bull World Health Organ 85: 441-8.

12. National Institutes of Health Consensus Development Conference on infantile apnea and home monitoring. Sept 29 to Oct 1, 1986. (1987) Pediatrics 79: 292-9.

13. Richards CA, Milla PJ, Andrews PL, Spitz L (2001) Retching and vomiting in neurologically impaired children after fundoplication: Predictive preoperative factors. J Pediatr Surg 36: 1401-4.

14. Hassall E (2005) Decisions in diagnosing and managing chronic gastroesophageal reflux disease in children. J Pedatr 146: S3-12.

15. Georgeson K (2002) Results of laparoscopic antireflux procedures in neurologically normal infants and children. Semin Laparosc Surg 9: 172-6.

16. Ngerncham M, Barnhart DC, Haricharna RN, Roseman JM, Georgeson KE, et al. (2007) Risk factors for recurrent reflux disease after fundoplication in pediatric patients: a case control study. J Pediatr Surg 42: 1478-85.

17. Pacilli M, Eaton S, Maritsi D, Lopez PJ, Spitz L, et al. (2007) Factors predicting failure of redo Nissen fundoplication in children. Pediatr Surg Int 23: 499-503.

18. Lee SL, Sydorak RM, Chiu VY, Hsu JW, Applebaum H, Haigh PI (2008) Long-term Antireflux Medication Use Following Pediatric Nissen Fundoplication. Arch Surg 143: 873-6.

19. Lopez-Fernandez S, Hernandez F, Hernandez-Martin S, Dominguez E, Ortiz R, et al. (2014) Failed Nissen fundoplication in children: causes and management. Euro J Pediatr Surg 24: 79-82.

20. Vadlamudi MB, Hitch MC, Dimmit RA, Thame KA (2013) Baclofen for the treatment of pediatric GERD. J Pediatr Gastroenterol Nutr 57: 808-12.

21. Wales P, Diamond I, Dutta S, Muraca S, Chait P, et al. (2002) Fundoplication and gastrostomy versus image-guided gastrojejunal tube for enteral feeding in neurologically impaired children with gastroesophageal reflux. J Pediatr Surg 37: 407-12. 
22. Lee SL, Shabatian H, Hsu JW, Applebaum H, Haigh PI (2008) Hospital admissions for respiratory symptoms and failure to thrive before and after Nissen fundoplication. J Pediatr Surg 43: 59-63.

23. Vaquero-Sosa E, Francisco-Gonzalez L, Bodas-Pinedo A, Urbasos-Garzon C, Ruiz-de-Leon-San-Juan A (2015) Oropharyngeal dysphagia, an underestimated disorder in pediatrics. Rev Esp Enferm Dig 107: 113-5.

24. Jobe BA, Richter JE, Hoppo T, Peters JH, Bell R (2013) Preoperative diagnostic workup before antireflux surgery: an evidence and experience based consesnsus of the esophageal diagnostic advisory panel. J Am Coll Surg 217: 586-97.

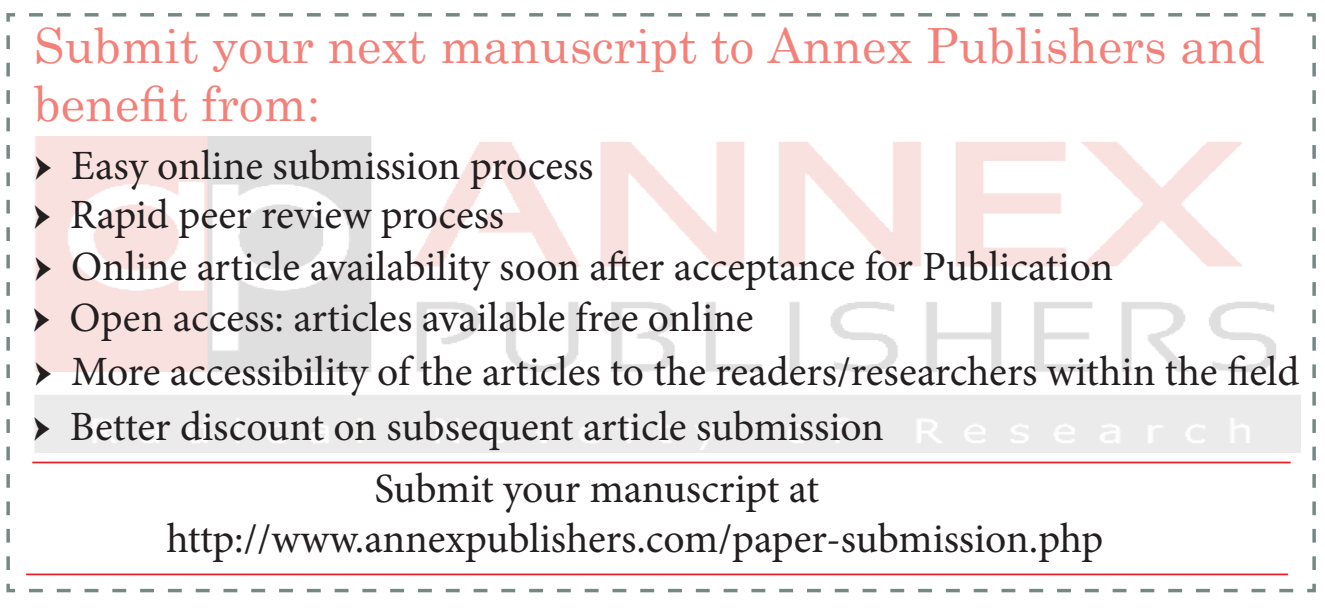

